\title{
ON BOUNDS OF HOMOLOGICAL DIMENSIONS IN NAKAYAMA ALGEBRAS
}

\author{
DAG OSKAR MADSEN AND RENÉ MARCZINZIK
}

(Communicated by Jerzy Weyman)

\begin{abstract}
Let $A$ be a Nakayama algebra with $n$ simple modules and a simple module $S$ of even projective dimension. Choose $m$ minimal such that a simple $A$-module with projective dimension $2 m$ exists. Then we show that the global dimension of $A$ is bounded by $n+m-1$. This gives a combined generalisation of results of Gustafson [J. Algebra 97 (1985), pp. 14-16] and Madsen [Projective dimensions and Nakayama algebras, Amer. Math. Soc., Providence, RI, 2005]. In [Comm. Algebra 22 (1994), pp. 1271-1280], Brown proved that the global dimension of quasi-hereditary Nakayama algebras with $n$ simple modules is bounded by $n$. Using our result on the bounds of global dimensions of Nakayama algebras, we give a short new proof of this result and generalise Brown's result from quasi-hereditary to standardly stratified Nakayama algebras, where the global dimension is replaced with the finitistic dimension.
\end{abstract}

\section{INTRODUCTION}

We always assume that our algebras are finite dimensional over a field $K$ and furthermore they are connected and nonsemisimple if nothing is stated otherwise. We assume that all modules are finite-dimensional right modules if nothing is stated otherwise. Nakayama algebras are defined as algebras such that every indecomposable projective left or right module is uniserial. See for example the books [ARS, [SY], and [im for sections on the basics and importance of Nakayama algebras.

In Gus, Gustafson showed that a Nakayama algebra with $n$ simple modules and finite global dimension has global dimension at most $2 n-2$, and in [Mad, Madsen showed that a Nakayama algebra has finite global dimension if and only if it has a simple module of even projective dimension. We combine and generalise those two results to the following, which is our first main result.

Theorem A. Let $A$ be a Nakayama algebra with $n$ simple modules and with a simple module of even projective dimension. Choose $m$ minimal such that a simple $A$-module has projective dimension equal to $2 \mathrm{~m}$. Then the global dimension of $A$ is bounded by $n+m-1$.

In forthcoming work we discuss whether the bounds $n+m-1$ are optimal and attained, where we discover a connection with the classification of Nakayama algebras that are higher Auslander algebras. Recall that the finitistic dimension findim $(A)$

Received by the editors October 17, 2017, and, in revised form, January 18, 2018.

2010 Mathematics Subject Classification. Primary 16G10, 16E10.

Key words and phrases. Global dimension, finitistic dimension, Nakayama algebras, standardly stratified algebras. 
of an algebra $A$ is defined as the supremum of all projective dimensions of modules having finite projective dimension. It is one of the most famous conjectures in the representation theory of finite-dimensional algebras that the finitistic dimension is always finite.

In [Bro, Brown showed that the global dimension of quasi-hereditary Nakayama algebras with $n$ simple modules is bounded by $n$. Standardly stratified algebras were introduced as a generalisation of quasi-hereditary algebras. Indeed, it was proven in AHLU that a standardly stratified algebra is quasi-hereditary if and only if it has finite global dimension. In recent years there was much interest in equalities and inequalities for the finitistic dimension of standardly stratified algebras; see for example [MO, AHLU2, [Mar2], and Maz.

We prove our second main result for standardly stratified Nakayama algebras.

Theorem B. Let $A$ be a standardly stratified Nakayama algebra with $n$ simple modules. Then $\operatorname{findim}(A) \leq n$.

The proof uses our first main result on the bounds of the global dimension to give a short proof for quasi-hereditary Nakayama algebras. We then classify the standardly stratified Nakayama algebras of infinite global dimension and look at their finitistic dimension to obtain our second main result. We remark that a proof of Proposition 3.2 was also obtained by Aaron Chan with more elementary methods.

\section{Preliminaries}

1.1. General preliminaries. Throughout $A$ is a finite-dimensional, nonsemisimple, and connected algebra over a field $K$. We always work with finite-dimensional right modules, if not stated otherwise. We denote by $\bmod -A$ the category of finitedimensional right $A$-modules and $J$ denotes the Jacobson radical of an algebra $A$. As usual, $D:=\operatorname{Hom}_{K}(-, K)$ denotes the $K$-duality of an algebra $A$ over the field $K$. For background on representation theory of finite-dimensional algebras and their homological algebra, we refer to [ARS] and [SY].

An algebra is called basic in case the regular module does not contain a projective module of the form $P^{2}$ as a direct summand for an indecomposable projective module $P$. Every algebra is Morita equivalent to a basic algebra and we thus assume that all our algebras are basic if nothing is stated otherwise. Note that all the homological notions in this text are invariant under Morita equivalence and thus it is no restriction on the generality of our results to assume that our algebras are basic. For a fixed set of primitive orthogonal idempotents $e_{1}, e_{2}, \ldots, e_{n}$ with $1=e_{1}+e_{2}+\cdots+e_{n}$, we denote by $S_{i}=e_{i} A / e_{i} J, P_{i}=e_{i} A$, and $I_{i}=D\left(A e_{i}\right)$ the simple, indecomposable projective, and indecomposable injective modules, respectively, corresponding to the primitive idempotent $e_{i}$, for $1 \leq i \leq n$. The global dimension is defined as the supremum of all projective dimensions of modules. Thus the global dimension coincides with the finitistic dimension in case the global dimension is finite.

An algebra $A$ is called a Nakayama algebra in case every indecomposable left or right module is uniserial. We refer to $\mathrm{AF}$ and $\mathrm{SY}$ ] for results on Nakayama algebras which we collect in the following without proof. A Nakayama algebra either has no simple projective module or it has a unique simple projective module. In the last case the algebra is triangular and hence the global dimension is bounded by $n-1$. If the Nakayama algebra is not triangular and has $n$ simple modules, it 
is possible to order the primitive idempotents such that there are projective covers

$$
e_{i+1} A \rightarrow e_{i} J
$$

for $1 \leq i \leq n-1$, and a projective cover

$$
e_{1} A \rightarrow e_{n} J
$$

Fix such an order $e_{1}, e_{2}, \ldots, e_{n}$ of primitive orthogonal idempotents. This order is uniquely defined up to a cyclic permutation. We also have $\tau\left(S_{i}\right) \cong S_{i+1}$, for $1 \leq i \leq n-1$, and $\tau\left(S_{n}\right) \cong S_{1}$, where $\tau$ denotes the Aulander-Reiten translate. The Nakayama algebra $A$ is uniquely determined by the length $c_{i}$ of the indecomposable projective modules $e_{i} A$. The sequence $\left[c_{1}, c_{2}, \ldots, c_{n}\right]$ is called the Kupisch series for $A$. One can show that $c_{i+1} \geq c_{i}-1$, for all $1 \leq i \leq n-1$, and $c_{1} \geq c_{n}-1$. Conversely, any sequence of integers greater than or equal to 2 satisfying those requirements is the Kupisch series for some Nakayama algebra. We look at the indices $i$ of the $c_{i}$ modulo $n$ so that $c_{i}$ is defined for all $i \in \mathbb{Z}$. A Nakayama algebra is self-injective if and only if its Kupisch series is constant. In case the algebra is not self-injective, then after a cyclic reordering of the indices one can always get $c_{1}=c_{n}-1$ with $c_{1}$ minimal among the $c_{i}$. Every indecomposable module of a Nakayama algebra is isomorphic to a module of the form $e_{i} A / e_{i} J^{k}$. For explicit calculations of minimal projective resolutions or injective coresolutions in Nakayama algebras, see for example $\mathrm{Mar}$.

The following lemma is a direct consequence of the classification of Nakayama algebras by their diagrams; see Chapter 10.3 of [DK] (note that in this textbook Nakayama algebras are called serial algebras). In Theorem 10.3.1 of [DK] it is proven that the diagram of a Nakayama algebra is either a directed line or a directed cycle.

Lemma 1.1. Let $A$ be a connected Nakayama algebra having no simple projective module, and let $e \in A$ be a primitive idempotent. Then $A / A e A$ is a connected Nakayama algebra of finite global dimension. The algebra eAe is also a connected Nakayama algebra which is semisimple if and only if the length of $e A$ as an $A$ module is less than or equal to $n$. If the algebra eAe is not semisimple, then $A / A e A$ is hereditary.

1.2. Preliminaries on standardly stratified algebras. See DR] for an introduction to quasi-hereditary algebras and Rei], ADL, and [FM] for the basics of standardly stratified algebras. We just briefly recall the most important definitions. Let $(A, E)$ be an algebra together with an ordered complete sequence of primitive orthogonal idempotents $E=\left(e_{1}, e_{2}, \ldots, e_{n}\right)$. Then the sequence of standard right $A$-modules is defined by $\Delta=(\Delta(1), \ldots, \Delta(n))$, where $\Delta(i)=$ $e_{i} A / e_{i} J\left(e_{i+1}+e_{i+2}+\cdots+e_{n}\right) A$ for $1 \leq i \leq n$ with $\Delta(n)=e_{n} A$. The sequence of proper standard right $A$-modules $\bar{\Delta}$ is defined by $\bar{\Delta}=(\bar{\Delta}(1), \ldots, \bar{\Delta}(n))$, where $\bar{\Delta}(i)=e_{i} A / e_{i} J\left(e_{i}+e_{i+1}+\cdots+e_{n}\right) A$ for $1 \leq i \leq n$. Dually, one can define left standard modules $\Delta^{o}(i)$ and left proper standard modules $\bar{\Delta}^{o}(i)$. The costandard modules $\nabla(i)$ and proper costandard modules $\bar{\nabla}(i)$ are then defined as the modules $D\left(\Delta^{o}(i)\right)$ and $D\left(\bar{\Delta}^{o}(i)\right)$. For a set of modules $C$, let $\mathcal{F}(C)$ be the full subcategory of mod- $A$ of all modules $M$ with a filtration $0 \subseteq M_{s} \subseteq \cdots \subseteq M_{1}=M$, such that every subquotient is isomorphic to an object in $C$. A module is called proper standardly filtered in case $M \in \mathcal{F}(\bar{\Delta})$ with $\bar{\Delta}:=\{\bar{\Delta}(1), \ldots, \bar{\Delta}(n)\}$. The algebra $A$ is called standardly stratified in case $A \in \mathcal{F}(\bar{\Delta})$. In view of [AHLU, Theorem 2.4], we define 
$A$ to be quasi-hereditary, in case it is standardly stratified and has finite global dimension. In case $A$ and $A^{\mathrm{op}}$ are both standardly stratified, then $A$ is called properly stratified (here we use a characterisation of properly stratified algebras found in Rei after Theorem 3.6).

We remark that if a Nakayama algebra is standardly stratified, the order of primitive idempotents defining the Kupisch series does not have to coincide with the sequence $E$ defining the standardly stratified structure.

The next proposition collects several results from the literature that we will need in this article.

Proposition 1.2. Let $A$ be a finite-dimensional algebra.

(1) Let e be a primitive idempotent of an algebra $A$ such that AeA is projective as a right $A$-module. In case an $A$-module $M$ has finite projective dimension, then the eAe-module $M e$ is projective.

(2) Let $A$ be a standardly stratified algebra. Then $A e_{n} A$ is projective as a right $A$-module, and $A / A e_{n} A$ is again standardly stratified.

(3) A Nakayama algebra with no simple projective module is quasi-hereditary if and only if there is a simple module of projective dimension equal to 2.

(4) Let $e$ be an idempotent such that AeA is projective as a right A-module, and let $X$ be an $A / A e A$-module. Then $\operatorname{pd}_{A}(X) \leq \operatorname{pd}_{A / A e A}(X)+1$.

(5) Let $e$ be an idempotent such that $A e A$ is projective as a right $A$-module and the algebra eAe is semisimple. Then $\operatorname{gldim}(A) \leq \operatorname{gldim}(A / A e A)+2$.

(6) Let $e$ be a primitive idempotent such that AeA is projective as a right $A$ module. Suppose $A / A e A$ has finitistic dimension equal to $k$. Then $A$ has finitistic dimension at most $k+2$.

Proof.

(1) See [AHLU2, Lemma 2.4].

(2) See the part above Proposition 1.1 in AHLU2.

(3) This is part of Proposition 3.1 of [UY].

(4) This is a special case of Lemma 5.8 of APT.

(5) This is a special case of Theorem 5.4 of [APT].

(6) See [AHLU2, Theorem 2.2].

\section{UPPER BOUNDS OF THE GLOBAL DIMENSION FOR NAKAYAMA ALGEBRAS}

In this section $A$ will always denote a Nakayama algebra.

Suppose $A$ has no simple projective module. Let $\mathcal{S}$ denote a complete set of representatives of the isomorphism classes of simple $A$-modules. Following [Mad, we define a function $\psi: \mathcal{S} \rightarrow \mathcal{S}$ by $\psi(S) \cong\left(\tau^{-1}\right)^{w(S)} S$, where $w(S)$ is the length of the injective envelope of $S$, for each $S \in \mathcal{S}$. We say that $S \in \mathcal{S}$ is $\psi$-regular if $\psi^{r}(S)=S$ for some $r \geq 1$. Dual definitions were earlier considered in Gus].

In $\mathrm{Mad}]$ we find the following criteria for finite global dimension.

Theorem 2.1 ([Mad, Theorem 3.3]). Let $A$ be a Nakayama algebra having no simple projective module. The following are equivalent.

(a) A has finite global dimension.

(b) The set of $\psi$-regular simple A-modules is exactly the set of simple A-modules with even projective dimension, and $\psi$ is a cyclic permutation on this set. 
(c) There is a simple A-module with even projective dimension.

Denote by $\mathcal{S}^{\psi} \subseteq \mathcal{S}$ the set of $\psi$-regular simple $A$-modules.

Theorem 2.2. Let $A$ be a Nakayama algebra with $n$ simple modules and with a simple module of even projective dimension. Choose $m$ minimal such that a simple $A$-module has projective dimension equal to $2 \mathrm{~m}$. Then the global dimension of $A$ is bounded by $n+m-1$.

Proof. If $m=0$, then $A$ is triangular and the global dimension is bounded by $n-1$.

Suppose $m>0$. By Theorem 2.1, the global dimension of $A$ is finite. We have

$$
\operatorname{gldim} A=\max _{S \in \mathcal{S}} \operatorname{id}(S)=\max _{S \in \mathcal{S}} \operatorname{pd}(S)
$$

and also

$$
\operatorname{gldim} A \leq \max _{S \in \mathcal{S}^{\psi}} \operatorname{pd}(S)+1 .
$$

This inequality follows from Theorem 2.1 and the fact that if the global dimension of $A$ is $g$, then there exist a simple module of projective dimension $g$ and a simple module of projective dimension $g-1$.

Let $d$ be the number of simple $A$-modules that are not $\psi$-regular. Then for any $S \in \mathcal{S}$ we have that $\psi^{d}(S)$ is $\psi$-regular, and hence by dualising the main argument from Gus, we get $\operatorname{id}(S) \leq 2 d$. So

$$
\operatorname{gldim} A \leq 2 d .
$$

Let $S^{\prime}$ be a simple module with $\operatorname{pd}\left(S^{\prime}\right)=2 m$. Then

$$
\mathcal{S}^{\psi}=\left\{S^{\prime}, \psi\left(S^{\prime}\right), \ldots, \psi^{n-d-1}\left(S^{\prime}\right)\right\} .
$$

It follows from repeated use of $[\mathrm{Mad}$, Proposition $3.2(\mathrm{~b})]$ that $\max _{S \in \mathcal{S}} \psi \operatorname{pd}(S) \leq$ $2 m+2(n-d-1)=2 m+2 n-2 d-2$. Hence

$$
\operatorname{gldim} A \leq 2 m+2 n-2 d-1 \text {. }
$$

Adding the two inequalities together, we get

$$
2 \cdot \operatorname{gldim} A \leq 2 m+2 n-1 .
$$

Since gldim $A$ must be an integer, we conclude that

$$
\operatorname{gldim} A \leq m+n-1 \text {. }
$$

We will discuss whether the bounds $n+m-1$ for given $m$ are attained in forthcoming work, where this is related to the classification of higher Auslander algebras with high global dimension inside the class of Nakayama algebras. We just give one example for $m=1$.

Example 2.3. Let $A$ be the Nakayama algebra with Kupisch series $[2,2,2, \ldots, 2,3]$, which has $n$ simple modules and all but one indecomposable projective module have length 2 . Then the simple module $S_{i}$ has projective dimension $n-i+1$, for $1 \leq i \leq n$, and hence the global dimension is $n$. The simple module $S_{n-1}$ has projective dimension 2 , and thus $m=1$. As a consequence, the bound $n+m-1$ is attained in case $m=1$. 


\section{Standardly Stratified NAKAyama Algebras AND THEIR FINITISTIC DIMENSION}

This section gives bounds on the finitistic dimension of standardly stratified Nakayama algebras. We can assume that all algebras involved are not self-injective as the next lemma shows. We note that the next lemma is a generalisation of the main result in $[\mathrm{AC}]$, where the authors proved the same result with the additional assumption that $A$ is a Nakayama algebra. We note that it seems that the authors in $\mathrm{AC}$ forgot to look at the local case, but their argument works with nearly the same proof in the general case.

Lemma 3.1. Let $A$ be a self-injective algebra. Then $A$ is standardly stratified if and only if $A$ is local. $A$ is never quasi-hereditary.

Proof. Since the first syzygy $\Omega^{1}$ is an equivalence on the stable module category $\underline{\bmod -A}$ (see for example section IV.8 in [SY]), every indecomposable nonprojective module $M$ has infinite projective dimension. But every standard module $\Delta(i)$, $1 \leq i \leq n$, has finite projective dimension by Proposition 1.3 of [PR], and thus every $\bar{\Delta}(i)$ is projective and hence also injective because $A$ is self-injective. By the definition of standard modules, $\Delta(i)=e_{i} A /\left(e_{i} J \epsilon_{i+1} A\right)$ with $\epsilon_{i+1}:=e_{i+1}+e_{i+2}+$ $\cdots+e_{n}$. Thus $\Delta(i)=e_{i} A /\left(e_{i} J \epsilon_{i+1} A\right)$ is projective for every $1 \leq i \leq n$ if and only if $\left(e_{i} J \epsilon_{i+1} A\right)=0$ for every $1 \leq i \leq n$.

Assume the algebra has at least two simple modules and take $i=1$. Then the condition $\left(e_{1} J \epsilon_{2} A\right)=0$ together with our assumption that $A$ is connected implies that $e_{1} J e_{i}=0$ for all $i>1$ and that there is a $j>1$ with $e_{j} J e_{1} \neq 0$. Now this implies that $e_{1} A$ has socle isomorphic to the socle of $D\left(A e_{1}\right)$ and also $e_{j} A$ has socle isomorphic to the socle of $D\left(A e_{1}\right)$. (Here the socle of indecomposable projective modules in a self-injective algebra is simple.) This is a contradiction, since a basic algebra is self-injective if and only if there is a permutation $\pi:\{1, \ldots, n\} \rightarrow\{1, \ldots, n\}$ such that $\operatorname{soc}\left(e_{i} A\right) \cong \operatorname{top}\left(e_{\pi(i)} A\right)$ for all $1 \leq i \leq n$. Thus $A$ has to be local.

On the other hand, assume now that $A$ is local and self-injective with simple module $S$. Then $\bar{\Delta}(1)=S$ and thus it is trivial that $A$ is $\bar{\Delta}$-filtered, since there is a unique simple module $S$. This implies that $A$ is standardly stratified. As a self-injective algebra, $A$ always has infinite global dimension and can thus never be quasi-hereditary. (Recall that we do assume that $A$ is not semisimple in our article.)

Since local Nakayama algebras are self-injective and local algebras in any case are of finitistic dimension 0 , we assume from now on that our algebras have at least two simple modules.

Proposition 3.2. Let $A$ be a connected Nakayama algebra having no simple projective module and having at least two simple modules. Let $A$ be standardly stratified but not quasi-hereditary, and assume that $A$ is not self-injective. Then there is a simple module of infinite projective dimension, and all other simple modules have projective dimension equal to 1.

Proof. Let $n$ denote the number of simple $A$-modules and assume that $A$ is standardly stratified. Let $e_{1}, e_{2}, \ldots, e_{n}$ be an ordering of the primitive idempotents for $A$ to be standardly stratified. By definition $\bar{\Delta}(n)=e_{n} A / e_{n} J e_{n} A$ and $\Delta(n)=e_{n} A$. By Proposition 1.2 (2), we have that $A e_{n} A$ is projective. Since $A$ is a Nakayama algebra, we have $e_{n} J e_{n} A=y A$ for an element $y \in e_{n} J e_{n} \backslash e_{n} J^{n+1} e_{n}$ (which is 
unique up to multiplication by a field element), and thus $\bar{\Delta}(n)=e_{n} A / y A$. We now look at two cases.

Case 1. Assume $\bar{\Delta}(n)=\Delta(n)$, which is equivalent to $e_{n} A / y A=e_{n} A$ or $y A=0$. This shows that $e_{n} J e_{n}=0$, and hence the algebra $e_{n} A e_{n}$ is semisimple. We can apply Proposition 1.2(5) and Lemma 1.1 to see that $A$ has finite global dimension and thus is quasi-hereditary.

Case 2. Now assume that $\bar{\Delta}(n) \neq \Delta(n)$, which is equivalent to $y A \neq 0$. We show that in this case the simple module $S_{n}$ has infinite projective dimension. Since $e_{n} y e_{n} \neq 0$, we have that the local algebra $e_{n} A e_{n}$ is not semisimple and thus a selfinjective Nakayama algebra, since for any Nakayama algebra $A, e A e$ is a Nakayama algebra again for any idempotent $e$. Let $M:=S_{n}$, and use Proposition 1.2(1) to see that $M$ has infinite projective dimension, or else the simple module $M e_{n}$ would be projective over the local self-injective connected algebra $e_{n} A e_{n}$, which is impossible. By Lemma 1.1, the algebra $A / A e_{n} A$ is hereditary. Now Proposition 1.2(4) gives that every simple $A$-module $S_{i}$ not isomorphic to $S_{n}$ has projective dimension at most 2. But such an $S_{i}$ cannot have projective dimension 0 by assumption on $A$ and not projective dimension 2 since then $A$ would be quasi-hereditary and thus have finite global dimension. So all simple $A$-modules except $S_{n}$ have projective dimension 1 , and this proves the proposition.

The following proposition gives the possible Kupisch series for Nakayama algebras that are standardly stratified but not quasi-hereditary.

Proposition 3.3. Let $A$ be a (nonself-injective) Nakayama algebra with $n$ simple modules and a simple module of infinite projective dimension and all other simple modules of projective dimension 1. Then the Kupisch series of $A$ is of the form

$[k+q n, k+n-1+q n, k+n-2+q n, k+n-3+q n, \ldots, k+2+q n, k+1+q n]$, where $2 \leq k \leq n$ and $q \geq 1$, or $k=n+1$ and $q \geq 0$.

Proof. Note that in a Nakayama algebra the simple module $S_{i}=e_{i} A / e_{i} J$ has projective dimension 1 if and only if $e_{i} J$ is projective if and only if $e_{i} J \cong e_{i+1} A$ if and only if $c_{i}-1=c_{i+1}$ in the Kupisch series $\left[c_{1}, c_{2}, \ldots, c_{n}\right]$ of $A$. Since $A$ has all but one simple module of projective dimension 1, the Kupisch series of $A$ has the form $[x, x+n-1, x+n-2, x+n-3, \ldots, x+1]$. Thus we can write the Kupisch series as stated. In case $q \geq 1$ or $k \geq n+1$, the Loewy length of the algebra is at least $2 n$, and thus the algebra has infinite global dimension by Gus] or alternatively by showing that in this case the first simple module has infinite projective dimension. In case $q=0$ and $k \leq n$, the first simple module has projective dimension 2 , and thus the algebra is quasi-hereditary by Proposition $1.2(3)$.

We give a corollary of the previous proposition.

Corollary 3.4. Let $A$ be a standardly stratified Nakayama algebra. Then $A$ is even properly stratified.

Proof. The result is clear in case $A$ is quasi-hereditary or self-injective, since the opposite algebra of a quasi-hereditary algebra is again quasi-hereditary in general and the opposite algebra of a local self-injective Nakayama algebra is again a local selfinjective Nakayama algebra. Now assume that $A$ is standardly stratified with infinite global dimension and not self-injective. Assume $A$ has $n$ simple modules. 
By Proposition 3.3 the Kupisch series of $A$ is of the form $[c, c+n-1, c+n-$ $2, \ldots, c+1]$ for some natural number $c \geq 2$. By Exercise 1 of Chapter 32 of [AF, the Kupisch series $\left[d_{1}, d_{2}, \ldots, d_{n}\right]$ of the opposite algebra of a Nakayama algebra with Kupisch series $\left[c_{1}, c_{2}, \ldots, c_{n}\right]$ has the property that the $d_{i}$ are a permutation of the $c_{i}$. As explained in the preliminaries we can assume that in a general Kupisch series we have $c_{n}=c_{1}+1$ with $c_{1}$ minimal among the $c_{i}$ and $c_{i+1} \geq c_{i}-1$. Those conditions, together with the fact that the Kupisch series of the opposite algebra is a permutation of the Kupisch series of $A$, force that the Kupisch series of the opposite algebra of $A$ coincides with the Kupisch series of $A$. Thus $A$ is isomorphic to its opposite algebra and the result is clear.

The next proposition shows that any Nakayama algebra with a Kupisch series as in Proposition 3.3 is indeed standardly stratified. Thus this gives a classification of the standardly stratified Nakayama algebras that are not quasi-hereditary. Since it is elementary to decide whether a Nakayama algebra with a given Kupisch series is standardly stratified or not and since we do not need the result in the following, we leave the proof of the next proposition to the interested reader.

Proposition 3.5. Let A be a Nakayama algebra with a Kupisch series as in Proposition 3.3. Then $A$ is standardly stratified.

We can now give a proof of our second main result.

Theorem 3.6. Let $A$ be a Nakayama algebra with $n$ simple modules that is standardly stratified. Then findim $A \leq n$. If $A$ is not quasi-hereditary, then findim $A \leq$ 2 .

Proof.

Case 1 (Quasi-hereditary case). If a Nakayama algebra has a simple projective module, then it is triangular and hence its global dimension is bounded by $n-1$.

By Proposition 1.2(3), we know that a Nakayama algebra having no simple projective module is quasi-hereditary if and only if there exists a simple module of projective dimension equal to 2. By Theorem 2.2. this implies that the global dimension, which is equal to the finitistic dimension, is bounded by $n+1-1=n$.

Case 2 (Infinite global dimension case). Now assume that our algebras are standardly stratified with infinite global dimension. Clearly, the result is trivial in the self-injective case, since self-injective algebras have finitistic dimension equal to 0 .

Now assume that the algebras are additionally nonself-injective. Then we use Proposition 3.3 to calculate the finitistic dimension of those algebras. Assume that the algebra has at least two simple modules. Let $e$ be a primitive idempotent such that $A e A$ is projective. In Proposition 3.3 we saw that each indecomposable projective module has length at least $n+1$, so $e A e$ is not semisimple by Lemma 1.1. Also by Lemma 1.1, the algebra $A / A e A$ is hereditary. Thus by Proposition 1.2(6), the finitistic dimension of $A$ is at most 3.

Let $M$ be an $A$-module with $\operatorname{pd}(M)=3$. From Proposition 2.2(a) in Mad it follows that all simple composition factors of $M$ have odd projective dimension, so they all have projective dimension 1 by Proposition 3.2. But a module cannot have larger projective dimension than the maximum pd of its composition factors, and we reach a contradiction. So in the infinite global dimension case findim $A \leq 2$. 
Example 3.7. Let $n \geq 2$. This example shows that the bounds in the previous theorem are optimal. First note that the algebra in Example 2.3 is a quasi-hereditary algebra with $n$ simple modules and global dimension $n$. Thus the finitistic dimension is equal to the global dimension since the global dimension is finite. This shows that the bound $n$ for the finitistic dimension is optimal. The Nakayama algebra with Kupisch series $[4,5]$ is standardly stratified with infinite global dimension. The unique indecomposable injective nonprojective module has projective dimension 2. Thus the bound 2 for finitistic dimensions of standardly stratified Nakayama algebras with infinite global dimension is also optimal.

\section{ACKNowledgments}

The second author thanks Aaron Chan for useful discussions. The authors profited from the GAP package QPA, see QPA, to calculate numerous examples.

\section{REFERENCES}

[AC] Advíncula, J.; Cruz, R.: Stratification analysis of certain Nakayama algebras. Advances in Pure Mathematics (2015), no. 5, 850-855.

[ADL] István Ágoston, Vlastimil Dlab, and Erzsébet Lukács, Stratified algebras (English, with French summary), C. R. Math. Acad. Sci. Soc. R. Can. 20 (1998), no. 1, 22-28. MR 1619048

[AHLU] István Ágoston, Dieter Happel, Erzsébet Lukács, and Luise Unger, Standardly stratified algebras and tilting, J. Algebra 226 (2000), no. 1, 144-160, DOI 10.1006/jabr.1999.8154. MR 1749881

[AHLU2] István Ágoston, Dieter Happel, Erzsébet Lukács, and Luise Unger, Finitistic dimension of standardly stratified algebras, Comm. Algebra 28 (2000), no. 6, 2745-2752, DOI 10.1080/00927870008826990. MR1757427

[AF] Frank W. Anderson and Kent R. Fuller, Rings and categories of modules, 2nd ed., Graduate Texts in Mathematics, vol. 13, Springer-Verlag, New York, 1992. MR.1245487

[APT] M. Auslander, M. I. Platzeck, and G. Todorov, Homological theory of idempotent ideals, Trans. Amer. Math. Soc. 332 (1992), no. 2, 667-692, DOI 10.2307/2154190. MR.1052903

[ARS] Maurice Auslander, Idun Reiten, and Sverre O. Smalø, Representation theory of Artin algebras, Corrected reprint of the 1995 original, Cambridge Studies in Advanced Mathematics, vol. 36, Cambridge University Press, Cambridge, 1997. MR 1476671

[Bro] Peter Brown, Global dimension of quasihereditary serial rings, Comm. Algebra 22 (1994), no. 4, 1271-1280, DOI 10.1080/00927879408824905. MR.1261259

[DR] Vlastimil Dlab and Claus Michael Ringel, The module theoretical approach to quasihereditary algebras, Representations of algebras and related topics (Kyoto, 1990), London Math. Soc. Lecture Note Ser., vol. 168, Cambridge Univ. Press, Cambridge, 1992, pp. 200-224. MR 1211481

[DK] Yurij A. Drozd and Vladimir V. Kirichenko, Finite-dimensional algebras, Translated from the 1980 Russian original and with an appendix by Vlastimil Dlab, SpringerVerlag, Berlin, 1994. MR.1284468

[FM] Anders Frisk and Volodymyr Mazorchuk, Properly stratified algebras and tilting, Proc. London Math. Soc. (3) 92 (2006), no. 1, 29-61, DOI 10.1017/S0024611505015431. MR2192384

[Gus] William H. Gustafson, Global dimension in serial rings, J. Algebra 97 (1985), no. 1, 14-16, DOI 10.1016/0021-8693(85)90069-9. MR812165

[Mad] Dag Madsen, Projective dimensions and Nakayama algebras, Representations of algebras and related topics, Fields Inst. Commun., vol. 45, Amer. Math. Soc., Providence, RI, 2005, pp. 247-265. MR2146655

[Mar] René Marczinzik, Upper bounds for the dominant dimension of Nakayama and related algebras, J. Algebra 496 (2018), 216-241, DOI 10.1016/j.jalgebra.2017.10.025. MR.3737839 
[Mar2] Marczinzik, R.: Auslander-Gorenstein algebras, standardly stratified algebras and dominant dimensions. arXiv:1610.02966 [math.RT].

[Maz] Volodymyr Mazorchuk, On finitistic dimension of stratified algebras, Algebra Discrete Math. 3 (2004), 77-88. MR2146106

[MO] Volodymyr Mazorchuk and Serge Ovsienko, Finitistic dimension of properly stratified algebras, Adv. Math. 186 (2004), no. 1, 251-265, DOI 10.1016/j.aim.2003.08.001. MR2065514

[PR] María Inés Platzeck and Idun Reiten, Modules of finite projective dimension for standardly stratified algebras, Comm. Algebra 29 (2001), no. 3, 973-986, DOI 10.1081/AGB100001660. MR1842391

[QPA] The QPA-team, QPA - Quivers, path algebras and representations - a GAP package, Version 1.25; 2016 (https://folk.ntnu.no/oyvinso/QPA/)

[Rei] Idun Reiten, Tilting theory and homologically finite subcategories with applications to quasihereditary algebras, Handbook of tilting theory, London Math. Soc. Lecture Note Ser., vol. 332, Cambridge Univ. Press, Cambridge, 2007, pp. 179-214, DOI 10.1017/CBO9780511735134.008. MR2384611

[SY] Andrzej Skowroński and Kunio Yamagata, Frobenius algebras I: Basic representation theory, EMS Textbooks in Mathematics, European Mathematical Society (EMS), Zürich, 2011. MR2894798

[UY] Morio Uematsu and Kunio Yamagata, On serial quasi-hereditary rings, Hokkaido Math. J. 19 (1990), no. 1, 165-174, DOI 10.14492/hokmj/1381517167. MR1039471

[Zim] Alexander Zimmermann, Representation theory, A homological algebra point of view, Algebra and Applications, vol. 19, Springer, Cham, 2014. MR.3289041

Faculty of education And arts, Nord University, Post Box 1490, NO-8049 Bodø, NorWAY

Email address: dag.o.madsen@nord.no

Institute of ALGebra AND Number theory, University of Stuttgart, Pfaffenwaldring 57, 70569 Stuttgart, Germany

Email address: marczire@mathematik.uni-stuttgart.de 